\title{
Some Connections between the Spherical and Parabolic Bases on the Cone Expressed in terms of the Macdonald Function
}

\author{
I. A. Shilin ${ }^{1,2}$ and Junesang $\mathrm{Choi}^{3}$ \\ ${ }^{1}$ Department of Mathematics, Sholokhov Moscow State University for the Humanities, Verkhnyaya Radishevskaya 16-18, \\ Moscow 109240, Russia \\ ${ }^{2}$ Department of Mathematical Modeling, Moscow Aviation Institute, Volokolamskoe Shosse 4, Moscow 125993, Russia \\ ${ }^{3}$ Department of Mathematics, Dongguk University, Gyeongju 780-714, Republic of Korea
}

Correspondence should be addressed to Junesang Choi; junesang@mail.dongguk.ac.kr

Received 7 November 2013; Accepted 26 December 2013; Published 11 February 2014

Academic Editor: Kwang Ho Shon

Copyright (C) 2014 I. A. Shilin and J. Choi. This is an open access article distributed under the Creative Commons Attribution License, which permits unrestricted use, distribution, and reproduction in any medium, provided the original work is properly cited.

Computing the matrix elements of the linear operator, which transforms the spherical basis of $S O(3,1)$-representation space into the hyperbolic basis, very recently, Shilin and Choi (2013) presented an integral formula involving the product of two Legendre functions of the first kind expressed in terms of ${ }_{4} F_{3}$-hypergeometric function and, using the general Mehler-Fock transform, another integral formula for the Legendre function of the first kind. In the sequel, we investigate the pairwise connections between the spherical, hyperbolic, and parabolic bases. Using the above connections, we give an interesting series involving the Gauss hypergeometric functions expressed in terms of the Macdonald function.

\section{Introduction and Preliminaries}

For completeness and an easier reference, we begin by just recalling some parts of $[1$, Section 1]. Let $\Lambda$ be the cone in the Euclidean space $\mathbb{R}^{4}$ defined by

$$
\Lambda:=\left\{\left(x_{1}, x_{2}, x_{3}, x_{4}\right) \in \mathbb{R}^{4} \mid x_{1}^{2}-x_{2}^{2}-x_{3}^{2}-x_{4}^{2}=0\right\},
$$

where $\mathbb{R}$ denotes the set of real numbers. Let $S O(3,1)$ be a multiplicative group consisting of all $4 \times 4$ matrices $g$ in $\mathbb{R}$ which satisfies the following two properties:

$$
\operatorname{det} g=1, \quad g^{T} I_{1,3} g=I_{1,3},
$$

where $g^{T}$ denotes (as usual) the transpose of the matrix $g$ and $I_{1,3}$ is a $4 \times 4$ matrix given by

$$
I_{1,3}:=\left(\begin{array}{cccc}
1 & 0 & 0 & 0 \\
0 & -1 & 0 & 0 \\
0 & 0 & -1 & 0 \\
0 & 0 & 0 & -1
\end{array}\right) .
$$

Remark 1. $S O(3,1)$ is called the special pseudoorthogonal group. $S O(3,1)$ is a group of linear operators preserving the quadratic form $x_{1}^{2}-x_{2}^{2}-x_{3}^{2}-x_{4}^{2}$, that is, the special classical Lorentz group. Similarly, for the group $S O(2,1)$, see [2-4].

For a $\sigma \in \mathbb{C}$ where $\mathbb{C}$ is the set of complex numbers, $\mathfrak{D}_{\sigma}$ denotes the linear space consisting of infinitely differentiable $\sigma$-homogeneous functions on $\Lambda$. The representation of $S O(3,1)$ in $\mathfrak{D}_{\sigma}$ is a homomorphism $g \mapsto T_{\sigma}(g)$, where the operator $T_{\sigma}(g)$ acts as $f(x) \mapsto f\left(g^{-1} x\right)$ in the space $\mathfrak{D}_{\sigma}$.

Let $\gamma_{1}$ be the intersection of $\Lambda$ and the hyperplane $x_{1}=1$, $\gamma_{2}$ the intersection of $\Lambda$ and the pair of hyperplanes $x_{4}= \pm 1$, and $\gamma_{3}$ the intersection of $\Lambda$ and the hyperplane $x_{1}+x_{4}=0$. In other words, $\gamma_{1}$ is a sphere with radius $\sqrt{2}, \gamma_{2}$ is a twosheet hyperboloid, and $\gamma_{3}$ is a paraboloid. It is seen that, for $i \in\{1,2,3\}, \gamma_{i}$ are two-parameter manifolds on $\Lambda$ explicitly given by

$$
\begin{array}{r}
\gamma_{1}=\left\{\left(1, \sin \alpha_{1} \sin \beta_{1}, \sin \alpha_{1} \cos \beta_{1}, \cos \alpha_{1}\right) \mid \alpha_{1} \in[0, \pi],\right. \\
\left.\beta_{1} \in[0,2 \pi)\right\},
\end{array}
$$


$\gamma_{2}=\left\{\left(\cosh \alpha_{2}, \sinh \alpha_{2} \sin \beta_{2}, \sinh \alpha_{2} \cos \beta_{2}, \pm 1\right) \mid \alpha_{2}>0\right.$,

$$
\left.\beta_{2} \in[0,2 \pi)\right\}
$$

$$
\gamma_{3}=\left\{\left(\frac{1+\alpha_{3}^{2}}{2}, \alpha_{3} \sin \beta_{3}, \alpha_{3} \cos \beta_{3}, \frac{1-\alpha_{3}^{2}}{2}\right) \mid \alpha_{3}>0\right. \text {, }
$$

$$
\left.\beta_{3} \in[0,2 \pi]\right\} \text {. }
$$

Let $H_{i}$ denote subgroups acting transitively on $\gamma_{i}(i=$ $1,2,3)$. It is noted, in particular, that $H_{1} \simeq S O(3)$ and $H_{2} \simeq S O(2,1)$, where $S O(n)$ is the group of rotations of $n$-dimensional Euclidean space $\mathbb{R}^{n}$ (for more details of this group, see [5, Chapter IX]). In order to describe the group $H_{3}$, in detail, on the linear space of all diagonal matrices $a:=\operatorname{diag}\left(a_{1}, a_{2}\right)$, we introduce a scalar product as $a \cdot b:=$ $\operatorname{tr}\left(a b^{T}\right)$. Then $\gamma_{3}$ is a homogeneous space of the subgroup $\mathrm{H}_{3}$ consisting of all matrices

$$
\left(\begin{array}{ccc}
1+\frac{1}{2}|a|^{2} & a & \frac{1}{2}|a|^{2} \\
a^{T} & \operatorname{diag}(1,1) & a^{T} \\
-\frac{1}{2}|a|^{2} & -a & 1-\frac{1}{2}|a|^{2}
\end{array}\right)
$$

where $|a|^{2}$ denotes the square of the length of the matrix $a$.

The $H_{1}$-invariant measure on $\gamma_{1}$ can be written as

$$
(\mathrm{d} x)_{\gamma_{1}}=\frac{\mathrm{d} x_{\tau(2)} \mathrm{d} x_{\tau(3)}}{\left|x_{\tau(4)}\right|}
$$

where $\tau$ is an arbitrary permutation of the set $\{2,3,4\}$. The $\mathrm{H}_{2}$-invariant measure on $\gamma_{2}$ is

$$
(\mathrm{d} x)_{\gamma_{2}}=\frac{\mathrm{d} x_{\tau(1)} \mathrm{d} x_{\tau(2)}}{\left|x_{\tau(3)}\right|} \quad\left(\tau \in \mathbf{S}_{3}\right)
$$

where $\mathbf{S}_{3}$ is the symmetric group. The $H_{3}$-invariant measure on $\gamma_{3}$ is

$$
(\mathrm{d} x)_{\gamma_{3}}=\mathrm{d} x_{2} \mathrm{~d} x_{3} .
$$

We define the bilinear functionals $\mathrm{F}_{j}: \mathfrak{D}_{\sigma} \times \mathfrak{D}_{\widehat{\sigma}} \rightarrow$ $\mathbb{C}(j=1,2,3)$ given by

$$
(u, v) \longmapsto \int_{\gamma_{j}} u(x) v(x)(\mathrm{d} x)_{\gamma_{j}}
$$

Then we observe the invariant property for the functionals $F_{j}$ asserted by the following lemma.

Lemma 2. If $\widehat{\sigma}=-\sigma-2$, then, for any $i, j \in\{1,2,3\}, \mathrm{F}_{i}=\mathrm{F}_{j}$.
Proof. Choose $\tau=\mathrm{id}$ in (6) and $\tau=\left(\begin{array}{lll}1 & 2 & 3\end{array}\right)$ in (7) and write the measures $(\mathrm{d} x)_{\gamma_{j}}(j \in\{1,2\})$ in $\left(\alpha_{j}, \beta_{j}\right)$-coordinate system, respectively:

$$
\begin{aligned}
& (\mathrm{d} x)_{\gamma_{1}}=\left|\frac{\partial\left(x_{2}, x_{3}\right)}{\partial\left(\alpha_{1}, \beta_{1}\right)}\right| \frac{\mathrm{d} \alpha_{1} \mathrm{~d} \beta_{1}}{\left|x_{4}\right|}=\sin \alpha_{1} \mathrm{~d} \alpha_{1} \mathrm{~d} \beta_{1}, \\
& (\mathrm{~d} x)_{\gamma_{2}}=\left|\frac{\partial\left(x_{2}, x_{3}\right)}{\partial\left(\alpha_{2}, \beta_{2}\right)}\right| \frac{\mathrm{d} \alpha_{2} \mathrm{~d} \beta_{2}}{\left|x_{1}\right|}=\sinh \alpha_{2} \mathrm{~d} \alpha_{2} \mathrm{~d} \beta_{2} .
\end{aligned}
$$

Here id and $\partial\left(x_{2}, x_{3}\right) / \partial\left(\alpha_{j}, \beta_{j}\right)$, respectively, denote the identical permutation and the corresponding Jacobian determinant.

Let $u \in \mathfrak{D}_{\sigma}$ and $v \in \mathfrak{D}_{-\sigma-2}$. Then

$\mathrm{F}_{2}(u, v)$

$$
\begin{aligned}
=\int_{\gamma_{2}} & {\left[\operatorname{sech} \alpha_{2}\right]^{2} u\left(1, \tanh \alpha_{2} \sin \beta_{2}, \tanh \alpha_{2} \cos \beta_{2}, \pm \operatorname{sech} \alpha_{2}\right) } \\
& \cdot v\left(1, \tanh \alpha_{2} \sin \beta_{2}, \tanh \alpha_{2} \cos \beta_{2}, \pm \operatorname{sech} \alpha_{2}\right) \\
& \times \sinh \alpha_{2} \mathrm{~d} \alpha_{2} \mathrm{~d} \beta_{2} .
\end{aligned}
$$

Setting $\cos \varphi:= \pm \operatorname{sech} \alpha_{2}$, we obtain $\mathrm{d} \alpha_{2}= \pm \cosh \alpha_{2} \mathrm{~d} \varphi$ and $\sinh \alpha_{2}=(1 / 2) \sin 2 \varphi$. We therefore find that

$$
\begin{aligned}
& \mathrm{F}_{2}(u, v) \\
& \quad=\int_{\gamma_{1}} u\left(1, \sin \varphi \sin \beta_{2}, \sin \varphi \cos \beta_{2}, \cos \varphi\right) \\
& \quad \cdot v\left(1, \sin \varphi \sin \beta_{2}, \sin \varphi \cos \beta_{2}, \cos \varphi\right) \sin \varphi \mathrm{d} \varphi \mathrm{d} \beta_{2},
\end{aligned}
$$

which implies $F_{2}=F_{1}$.

In the same way,

$$
\begin{aligned}
& \mathrm{F}_{3}(u, v) \\
&=4 \int_{\gamma_{3}}\left(1+\alpha_{3}^{2}\right)^{-2} u\left(1, \frac{2 \alpha_{3} \sin \beta_{3}}{1+\alpha_{3}^{2}}, \frac{2 \alpha_{3} \cos \beta_{3}}{1+\alpha_{3}^{2}}, \frac{1-\alpha_{3}^{2}}{1+\alpha_{3}^{2}}\right) \\
& \cdot v\left(1, \frac{2 \alpha_{3} \sin \beta_{3}}{1+\alpha_{3}^{2}}, \frac{2 \alpha_{3} \cos \beta_{3}}{1+\alpha_{3}^{2}}, \frac{1-\alpha_{3}^{2}}{1+\alpha_{3}^{2}}\right) \alpha_{3} \mathrm{~d} \alpha_{3} \mathrm{~d} \beta_{3} .
\end{aligned}
$$

Setting here $\cos \varphi:=\left(1-\alpha_{3}^{2}\right) /\left(1+\alpha_{3}^{2}\right)$, we obtain $\sin \varphi=$ $2 \alpha_{3}^{2} /\left(1+\alpha_{3}^{2}\right)$ and $\mathrm{d} \alpha_{3}=\left(\left(1+\alpha_{3}^{2}\right) \mathrm{d} \varphi\right) / 2$. It means that

$$
\begin{aligned}
& \mathrm{F}_{3}(u, v) \\
& =\int_{\gamma_{1}} u\left(1, \sin \varphi \sin \beta_{3}, \sin \varphi \cos \beta_{3}, \cos \varphi\right) \\
& \quad \cdot v\left(1, \sin \varphi \sin \beta_{3}, \sin \varphi \cos \beta_{3}, \cos \varphi\right) \sin \varphi \mathrm{d} \varphi \mathrm{d} \beta_{3} .
\end{aligned}
$$

We thus see that $F_{1}=F_{3}$.

Since the relation $=$ defined on the set $\left\{F_{1}, F_{2}, F_{3}\right\}$ is transitive, we have $F_{2}=F_{3}$. The proof is complete. 
In [5, Chapter IX], Vilenkin constructed the canonical basis $\Xi_{K}^{l}$ on a sphere. Here, continuing this canonical basis from the sphere $\gamma_{1}$ to the cone $\Lambda$ via $\sigma$-homogeneity, we obtain the basis consisting of $f_{p_{1}, q_{1}}$ :

$$
\begin{aligned}
& f_{p_{1}, q_{1}}(x) \\
& =x_{1}^{\sigma-\left|q_{1}\right|} C_{p_{1}-\left|q_{1}\right|}^{\left|q_{1}\right| \mid((1 / 2)}\left(\frac{x_{4}}{x_{1}}\right)\left(x_{3}+\mathbf{i} x_{2} \operatorname{sign} q_{1}\right)^{\left|q_{1}\right|} \\
& \quad\left(\mathbf{i}=\sqrt{-1} ; \quad p_{1}, q_{1} \in \mathbb{Z} ; p_{1} \geq 0,\left|q_{1}\right| \leq p_{1}\right),
\end{aligned}
$$

where $\mathbb{Z}$ denotes the set of integers and $C_{m}^{p}(t)$ are the Gegenbauer polynomials (see, e.g., [6, Chapter 17]; see also [5, page 458, Equation (3)]). We will call it the spherical basis. Similarly, the hyperbolic basis consists of functions of the following forms:

$$
\begin{aligned}
f_{p_{2}, q_{2}, \pm}^{*} & (x) \\
= & \left(x_{4}\right)_{ \pm}^{\sigma}\left(x_{2}^{2}+x_{3}^{2}\right)^{-\left|q_{2}\right| / 2} P_{-(1 / 2)+\mathbf{i} p_{2}}^{-\left|q_{2}\right|}\left(\frac{x_{1}}{\left(x_{4}\right)_{ \pm}}\right) \\
& \times\left(x_{3}+\mathbf{i} x_{2} \operatorname{sign} q_{2}\right)^{\left|q_{2}\right|} \quad\left(p_{2}>0, q_{2} \in \mathbb{Z}\right),
\end{aligned}
$$

where $P_{\nu}^{\mu}(z)$ are the Legendre functions of the first kind (see, e.g., $[7$, pages $194-228]$; see also $[8,9])$ and

$$
(x)_{ \pm}^{\mu}= \begin{cases}0, & \text { if } \operatorname{sign} x=\mp 1 \text { or } x=0, \\ |x|^{\mu}, & \text { if sign } x= \pm 1\end{cases}
$$

About the nonhomogeneous hyperbolic part of the hyperbolic basis, see, for instance, [5, Chapter X]. Finally, the parabolic basis consists of the functions

$$
\begin{aligned}
f_{p_{3}, q_{3}}^{* *}(x) & \\
:= & \left(x_{1}+x_{4}\right)^{\sigma}\left(x_{2}^{2}+x_{3}^{2}\right)^{-\left|q_{3}\right| / 2} J_{\left|q_{3}\right|}\left(\frac{\sqrt{x_{2}^{2}+x_{3}^{2}} p_{3}}{x_{1}+x_{4}}\right) \\
& \times\left(x_{2}+\mathbf{i} x_{2} \operatorname{sign} q_{3}\right)^{\left|q_{3}\right|},
\end{aligned}
$$

where $p_{3} \geq 0, q_{3} \in \mathbb{Z}$, and $J_{v}(z)$ are the Bessel functions. The main idea of this basis is described in [5, Chapters IV and XI]; the multiplier $\left(x_{1}+x_{4}\right)^{\sigma}$ is its $\sigma$-homogeneous part.

Computing the matrix elements of the linear operator, which transforms the spherical basis of $S O(3,1)$ representation space into the hyperbolic basis, very recently, Shilin and Choi [1] presented an integral formula involving the product of two Legendre functions of the first kind expressed in terms of ${ }_{4} F_{3}$-hypergeometric function and, using the general Mehler-Fock transform (see, e.g., [10, 11]), another integral formula for the Legendre function of the first kind. In the sequel, using the matrix elements of the linear operators, which transform the spherical basis into the parabolic basis and the hyperbolic basis into the parabolic basis, respectively, here, in this paper, we also give certain connections between the spherical and parabolic bases on the cone and an interesting series involving the Gauss hypergeometric functions, both expressed in terms of the Macdonald function.

\section{Description of the Connection between the Spherical and Hyperbolic Bases in terms of ${ }_{4} F_{3}$ Function}

Using

$$
\int_{0}^{2 \pi} \exp (\mathbf{i}(p+q) z) \mathrm{d} z=2 \pi \delta_{p,-q}
$$

and the orthogonality relation (see, e.g., [6, page 281, Equations (27) and (28)]; see also [5, page 462, Equations (4) and (5)])

$$
\begin{gathered}
\int_{-1}^{1} C_{p}^{q}(z) C_{\tilde{p}}^{q}(z)\left(1-z^{2}\right)^{q-(1 / 2)} \mathrm{d} z \\
=\frac{\pi \Gamma(2 q+p) \delta_{p, \tilde{p}}}{2^{2 q-1} p !(p+q)[\Gamma(q)]^{2}},
\end{gathered}
$$

where $\delta_{i, j}$ is the Kronecker symbol, we find from the decomposition

$$
f_{p_{2}, q_{2}, \pm}^{*}(x)=\sum_{p_{1}=0}^{\infty} \sum_{l=-\left|p_{1}\right|}^{\left|p_{1}\right|} c_{\left(p_{2}, q_{2},+\right),\left(p_{1}, l\right)} f_{p_{1}, l}(x)
$$

that

$$
\begin{aligned}
\mathcal{c}_{\left(p_{2}, q_{2}, \pm\right),\left(p_{1}, l\right)} & \\
= & \frac{2^{2 l-1}\left(p_{1}-l\right) !\left(p_{1}+(1 / 2)\right)[\Gamma(l+(1 / 2))]^{2}}{\pi^{2} \Gamma(2 l+1)} \\
& \times \mathrm{F}_{j}\left(f_{p_{2}, q_{2},+}^{*}, f_{p_{1},-l}\right),
\end{aligned}
$$

where $\Gamma$ is the familiar Gamma function (see, e.g., [12, Section 1.1]). Shilin and Choi [1] then observed that the numbers $\mathrm{F}_{j}\left(f_{p_{2}, q_{2}, \pm}^{*}, f_{p_{1}, q_{1}}\right)$ give a relationship between the spherical and hyperbolic bases asserted by the following theorem.

Theorem 3. Each of the following formulas holds true:

$$
\mathrm{F}_{2}\left(f_{p_{2}, q_{2},+}^{*}, f_{p_{1}, q_{1}}\right)=0 \quad\left(q_{1} \neq-q_{2}\right)
$$

and, for $\mathfrak{R}(\sigma)<-(1 / 2)$, 


$$
\begin{aligned}
\mathrm{F}_{2}\left(f_{p_{2},-q_{1},+}^{*}, f_{p_{1}, q_{1}}\right)= & 2^{p_{1}-\left|q_{1}\right|-\sigma-1} \sqrt{\pi} \\
& \cdot \frac{\Gamma\left((1 / 2)+p_{1}\right) \Gamma\left(\left(\left(p_{1}-\left|q_{1}\right|-\sigma+\mathbf{i} p_{2}\right) / 2\right)-(1 / 4)\right) \Gamma\left(\left(\left(p_{1}-\left|q_{1}\right|-\sigma-\mathbf{i} p_{2}\right) / 2\right)-(1 / 4)\right)}{\Gamma\left(1+p_{1}-\left|q_{1}\right|\right) \Gamma\left(p_{1}-\sigma\right) \Gamma\left((1 / 2)+\left|q_{1}\right|\right)} \\
& \cdot{ }_{4} F_{3}\left[\begin{array}{l}
\frac{\left|q_{1}\right|-p_{1}}{2}, \frac{1+\left|q_{1}\right|-p_{1}}{2}, \frac{1+\sigma-p_{1}}{2}, 1-\frac{\sigma+p_{1}}{2} ; \\
\frac{1}{2}-p_{1}, \frac{5}{4}+\frac{\sigma+\left|q_{1}\right|-p_{1}+\mathbf{i} p_{2}}{2}, \frac{5}{4}+\frac{\sigma+\left|q_{1}\right|-p_{1}-\mathbf{i} p_{2}}{2} ;
\end{array}\right],
\end{aligned}
$$

provided that one of the numerator parameters in ${ }_{4} F_{3}$ is zero or a negative integer, and ${ }_{p} F_{q}$ are the generalized hypergeometric functions (see, e.g., [12, Section 1.5]).

Here we provide a more general result than the one in Theorem 3 for all matrix elements $c_{\left(p_{2}, q_{2}, \pm\right),\left(p_{1}, q_{1}\right)}$ instead of $c_{\left(p_{2}, q_{2},+\right),\left(p_{1}, q_{1}\right)}$. We omit the corresponding proof of Theorem 3 to reformulate Theorem 3 for a general case asserted by Theorem $1^{*}$.

$$
\begin{aligned}
\mathrm{F}_{2}\left(f_{p_{2},-q_{1}, l}^{*}, f_{p_{1}, q_{1}}\right)= & \Psi_{\iota, p_{1}, q_{1}} 2^{p_{1}-\left|q_{1}\right|-\sigma-1} \sqrt{\pi} \\
& \cdot \frac{\Gamma\left((1 / 2)+p_{1}\right) \Gamma\left(\left(\left(p_{1}-\left|q_{1}\right|-\sigma+\mathbf{i} p_{2}\right) / 2\right)-(1 / 4)\right) \Gamma\left(\left(\left(p_{1}-\left|q_{1}\right|-\sigma-\mathbf{i} p_{2}\right) / 2\right)-(1 / 4)\right)}{\Gamma\left(1+p_{1}-\left|q_{1}\right|\right) \Gamma\left(p_{1}-\sigma\right) \Gamma\left((1 / 2)+\left|q_{1}\right|\right)} \\
& \cdot{ }_{4} F_{3}\left[\begin{array}{c}
\frac{\left|q_{1}\right|-p_{1}}{2}, \frac{1+\left|q_{1}\right|-p_{1}}{2}, \frac{1+\sigma-p_{1}}{2}, 1-\frac{\sigma+p_{1}}{2} ; \\
\frac{1}{2}-p_{1}, \frac{5}{4}+\frac{\sigma+\left|q_{1}\right|-p_{1}+\mathbf{i} p_{2}}{2}, \frac{5}{4}+\frac{\sigma+\left|q_{1}\right|-p_{1}-\mathbf{i} p_{2}}{2} ;
\end{array}\right],
\end{aligned}
$$

Theorem $1^{*}$. Let

$$
\Psi_{\iota, p_{1}, q_{1}}:= \begin{cases}1, & \text { if } \iota=+ \text { or } p_{1}-\left|q_{1}\right| \text { is even, } \\ -1, & \text { if } \iota=-, p_{1}-\left|q_{1}\right| \text { is odd. }\end{cases}
$$

Then each of the following formulas holds true:

$$
\mathrm{F}_{2}\left(f_{p_{2}, q_{2}, \pm}^{*}, f_{p_{1}, q_{1}}\right)=0 \quad\left(q_{1} \neq-q_{2}\right)
$$

and, for $\mathfrak{R}(\sigma)<-(1 / 2)$, provided that one of the numerator parameters in ${ }_{4} F_{3}$ is zero or a negative integer, and ${ }_{p} F_{q}$ are the generalized hypergeometric functions (see, e.g., [12, Section 1.5]).

\section{Description of the Connection between the Spherical and Parabolic Bases in terms of the Macdonald Function}

Using the same method used in Section 2, from the decomposition

$$
f_{p_{3}, q_{3}}^{* *}(x)=\sum_{p_{1}=0}^{\infty} \sum_{q_{1}=-\left|p_{1}\right|}^{\left|p_{1}\right|} d_{\left(p_{3}, q_{3}\right),\left(p_{1}, q_{1}\right)} f_{p_{1}, q_{1}}(x),
$$

we derive

$$
\begin{aligned}
& d_{\left(p_{3}, q_{3}\right),\left(p_{1}, q_{1}\right)} \\
& =\frac{2^{2\left|q_{1}\right|-1}\left(p_{1}-\left|q_{1}\right|\right) !\left(p_{1}+(1 / 2)\right)\left[\Gamma\left(\left|q_{1}\right|+(1 / 2)\right)\right]^{2}}{\pi^{2} \Gamma\left(2\left|q_{1}\right|+1\right)} \\
& \quad \times \mathrm{F}_{j}\left(f_{p_{3}, q_{3}}^{* *}, f_{p_{1},-\left|q_{1}\right|}\right) .
\end{aligned}
$$

Theorem 4. The following formula holds true: for $\mathfrak{R}(\sigma)<$ $-(1 / 4)$,

$$
\begin{aligned}
\mathrm{F}_{3} & \left(f_{p_{3}, q_{3}}^{* *}, f_{\left|q_{3}\right|,-q_{3}}\right) \\
& =4 \pi p_{3}^{\left|q_{3}\right|-\sigma-1}\left[\Gamma\left(\left|q_{3}\right|-\sigma\right)\right]^{-1} K_{\sigma+1}\left(p_{3}\right),
\end{aligned}
$$

where $K_{\sigma+1}\left(p_{3}\right)$ is the Macdonald function (the modified Bessel function of the second kind) (see, e.g., [13, page 675]).

Proof. We have

$$
\begin{aligned}
& \mathrm{F}_{3}\left(f_{p_{3}, q_{3}}^{* *}, f_{p_{1},-q_{3}}\right) \\
& =2^{\left|q_{3}\right|-\sigma+1} \pi \\
& \quad \cdot \int_{0}^{+\infty} \alpha_{3}^{\left|q_{3}\right|+1}\left(1+\alpha_{3}^{2}\right)^{\sigma-\left|q_{3}\right|} J_{\left|q_{3}\right|}\left(p_{3} \alpha_{3}\right) \\
& \quad \times C_{p_{1}-\left|q_{3}\right|}^{\left|q_{3}\right|+(1 / 2)}\left(\frac{1-\alpha_{3}^{2}}{1+\alpha_{3}^{2}}\right) \mathrm{d} \alpha_{3} .
\end{aligned}
$$


Using the explicit representation (see, e.g., [14, page 175, Equation (18)])

$$
C_{n}^{v}(x)=\frac{1}{\Gamma(\nu)} \sum_{j=0}^{[n / 2]} \frac{(-1)^{j} \Gamma(n+\nu-j)}{j !(n-2 j) !}(2 x)^{n-2 j}
$$

$[u]$ being the greatest integer less than or equal to $u$, we obtain

$$
\begin{aligned}
& \mathrm{F}_{3}\left(f_{p_{3}, q_{3}}^{* *}, f_{p_{1},-q_{3}}\right) \\
& =2^{p_{1} \sigma+1} \pi\left[\Gamma\left(\left|q_{3}\right|+\frac{1}{2}\right)\right]^{-1} \\
& \quad \times \sum_{j=0}^{\left[\left(p_{1}-\left|q_{3}\right|\right) / 2\right]} \frac{(-1)^{j} 2^{-2 j} \Gamma\left(p_{1}-j+(1 / 2)\right)}{j !\left(p_{1}-\left|q_{3}\right|-2 j\right) !} \\
& \quad \cdot \int_{0}^{+\infty} \alpha_{3}^{\left|q_{3}\right|+1}\left(1+\alpha_{3}^{2}\right)^{\sigma-p_{1}+2 j} \\
& \quad \times\left(1-\alpha_{3}^{2}\right)^{p_{1}-\left|q_{3}\right|-2 j} J_{\left|q_{3}\right|}\left(p_{3} \alpha_{3}\right) \mathrm{d} \alpha_{3} .
\end{aligned}
$$

In case of $p_{1}=\left|q_{3}\right|$, we can use the following known formula (see [15, page 179, Entry 2.12.4-28]):

$$
\int_{0}^{+\infty} \frac{x^{\nu+1}}{\left(x^{2}+z^{2}\right)^{\rho}} J_{v}(c x) \mathrm{d} x=\frac{c^{\rho-1} z^{\nu-\rho+1}}{2^{\rho-1} \Gamma(\rho)} K_{\nu-\rho+1},
$$

which holds for $c>0, \mathfrak{R}(z)>0$, and $-1<\mathfrak{R}(\nu)<2 \mathfrak{R}(\rho)-$ $(1 / 2)$.

Corollary 5. The following formula holds true: for $\mathfrak{R}(\sigma)<$ $-(1 / 4)$,

$$
\begin{aligned}
& d_{\left(p_{3}, q_{3}\right),\left(\left|q_{3}\right|,-q_{3}\right)} \\
& =\left(\left(2^{2\left|q_{3}\right|+1} p_{3}^{\left|q_{3}\right|-\sigma-1}\left(\left|q_{3}\right|+\frac{1}{2}\right)\left[\Gamma\left(\left|q_{3}\right|+\frac{1}{2}\right)\right]^{2}\right)\right. \\
& \left.\quad \times\left(\pi \Gamma\left(\left|q_{3}\right|-\sigma\right) \Gamma\left(2\left|q_{3}\right|+1\right)\right)^{-1}\right) \\
& \quad \times K_{\sigma+1}\left(p_{3}\right) .
\end{aligned}
$$

Further we will deal only with the matrix elements $d_{\left(p_{3}, q_{3}\right),\left(p_{1},-q_{3}\right)}$ in case of $p_{1}=\left|q_{3}\right|$, but let us pay attention to general case. Let $\mathfrak{R}(\sigma)<-(1 / 4)$. As

$$
\begin{aligned}
p_{1} & -2 j-\frac{\left|q_{3}\right|}{2}-k-\frac{1}{4} \\
& =\left(p_{1}-\left|q_{3}\right|-2 j-k\right)+\frac{\left|q_{3}\right|}{2}-\frac{1}{4} \geq-\frac{1}{4},
\end{aligned}
$$

by using the binomial theorem for the factor $\left(1-\alpha_{3}^{2}\right)^{p_{1}-\left|q_{3}\right|-2 j}$ in (33) and formula (34), apparently, we can represent numbers $\mathrm{F}_{3}\left(f_{p_{3}, q_{3}}^{* *}, f_{p_{1},-q_{3}}\right)$ as a sum whose terms contain a product of the Macdonald function and the generalized hypergeometric function. Without loss of generality, we demonstrate it, for example, under condition $p_{1}-\left|q_{3}\right|=4$. In this case,

$$
\mathrm{F}_{3}\left(f_{p_{3}, q_{3}}^{* *}, f_{p_{1},-q_{3}}\right)=\frac{4 \pi p_{3}^{p_{1}-\sigma-1}}{\Gamma\left(\left|q_{3}\right|+(1 / 2)\right)} \sum_{j=0}^{\left[\left(p_{1}-\left|q_{3}\right|\right) / 2\right]} \Xi_{j} \cdot \Theta_{j}
$$

where

$$
\begin{gathered}
\Xi_{j}=\frac{(-1)^{j} p_{3}^{-2 j} \Gamma\left(p_{1}-j+(1 / 2)\right)}{j !\left(p_{1}-\left|q_{3}\right|-2 j\right) ! \Gamma\left(p_{1}-\sigma-2 j\right)}, \\
\Theta_{j}=\sum_{k=1}^{p_{1}-\left|q_{3}\right|-2 j}(-1)^{k}\left(\begin{array}{c}
p_{1}-\left|q_{3}\right|-2 j \\
k
\end{array}\right) K_{\sigma-(4-2 j)+2 k+1}\left(p_{3}\right) .
\end{gathered}
$$

As

$$
\begin{gathered}
\Gamma\left(p_{1}-j+(1 / 2)\right)=\frac{(-1)^{j} \Gamma\left(p_{1}+(1 / 2)\right)}{\left((1 / 2)-p_{1}\right)_{j}}, \\
\Gamma\left(p_{1}-\sigma-2 j\right) \\
=\frac{2^{-2 j} \Gamma\left(p_{1}-\sigma\right)}{\left(\left(1+\sigma-p_{1}\right) / 2\right)_{j}\left(1-\left(\left(\sigma+p_{1}\right) / 2\right)\right)_{j}},
\end{gathered}
$$

$$
\sum_{k=0}^{n}\left(\begin{array}{l}
n \\
k
\end{array}\right) K_{\nu \pm 2 k}(z)=(-2)^{n} K_{\nu \pm n}(z)
$$

(regarding the last formula see, e.g., [15, Entry 4.2.4-2]), we have

$$
\begin{aligned}
& \Theta_{0}= K_{\sigma+1}\left(p_{3}\right)+8\left(K_{\sigma-1}\left(p_{3}\right)+K_{\sigma+3}\left(p_{3}\right)\right) \\
&= 17 K_{\sigma+1}\left(p_{3}\right)+\frac{2 \sigma}{p_{3}}\left(K_{\sigma+2}\left(p_{3}\right)-K_{\sigma}\left(p_{3}\right)\right) \\
&+\frac{4}{p_{3}} K_{\sigma+2}\left(p_{3}\right) \\
&=\left(17+\frac{4 \sigma(\sigma+1)}{p_{3}^{2}}\right) K_{\sigma+1}\left(p_{3}\right)+\frac{4}{p_{3}} K_{\sigma+2}\left(p_{3}\right), \\
& \Theta_{1}= \frac{2(\sigma+2)}{p_{3}} K_{\sigma+2}\left(p_{3}\right)-\frac{2 \sigma}{p_{3}} K_{\sigma}\left(p_{3}\right) \\
&= \frac{4 \sigma(\sigma+1)}{p_{3}^{2}} K_{\sigma+1}\left(p_{3}\right)+\frac{4}{p_{3}} K_{\sigma+2}\left(p_{3}\right) \\
& \Theta_{2}=K_{\sigma+1}\left(p_{3}\right)
\end{aligned}
$$


and, therefore,

$$
\begin{aligned}
& \mathrm{F}_{3}\left(f_{p_{3}, q_{3}}^{* *}, f_{p_{1},-q_{3}}\right) \\
& =\frac{4 \pi p_{3}^{p_{1}-\sigma-1}}{\Gamma\left(\left|q_{3}\right|+(1 / 2)\right)} \\
& \cdot\left(\frac{17 \Gamma\left((1 / 2)+p_{1}\right)}{\Gamma\left(1+p_{1}-\left|q_{3}\right|\right) \Gamma\left(p_{1}-\sigma\right)}\right. \\
& \times K_{\sigma+1}\left(p_{3}\right) \sum_{j=0}^{\infty}(-1)_{j} \Xi_{j} \\
& +\frac{4 \sigma(\sigma+1) \Gamma\left((1 / 2)+p_{1}\right)}{p_{3}^{2} \Gamma\left(1+p_{1}-\left|q_{3}\right|\right) \Gamma\left(p_{1}-\sigma\right)} \\
& \mathrm{F}_{3}\left(f_{p_{3}, q_{3}}^{* *}, f_{p_{1},-q_{3}}\right)=\frac{4 \pi p_{3}^{p_{1}-\sigma-1}}{\Gamma\left(\left|q_{3}\right|+\frac{1}{2}\right)} \\
& \cdot\left(\frac{17 \Gamma\left((1 / 2)+p_{1}\right)}{\Gamma\left(1+p_{1}-\left|q_{3}\right|\right) \Gamma\left(p_{1}-\sigma\right)} K_{\sigma+1}\left(p_{3}\right)\right.
\end{aligned}
$$

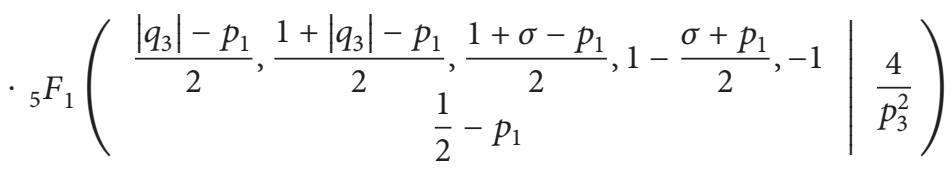

$$
\begin{aligned}
& +\frac{4 \sigma(\sigma+1) \Gamma\left((1 / 2)+p_{1}\right)}{p_{3}^{2} \Gamma\left(1+p_{1}-\left|q_{3}\right|\right) \Gamma\left(p_{1}-\sigma\right)} K_{\sigma+1}\left(p_{3}\right) \\
& \cdot{ }_{5} F_{2}\left(\begin{array}{c|c|c}
\frac{\left|q_{3}\right|-p_{1}}{2}, \frac{1+\left|q_{3}\right|-p_{1}}{2}, \frac{1+\sigma-p_{1}}{2}, 1-\frac{\sigma+p_{1}}{2},-2 & \frac{4}{p_{3}^{2}}
\end{array}\right) \\
& +\frac{\Gamma\left(p_{1}-(3 / 2)\right)}{p_{3}^{4} \Gamma\left(p_{1}-\left|q_{3}\right|-3\right) \Gamma\left(p_{1}-\sigma-4\right)} K_{\sigma+1}\left(p_{3}\right) \\
& \cdot{ }_{5} F_{2}\left(\begin{array}{c|c|c}
2+\frac{\left|q_{3}\right|-p_{1}}{2}, \frac{5+\left|q_{3}\right|-p_{1}}{2}, \frac{5+\sigma-p_{1}}{2}, 3-\frac{\sigma+p_{1}}{2},-1 & \frac{4}{p_{3}^{2}}
\end{array}\right) \\
& +\frac{4 \Gamma\left((1 / 2)+p_{1}\right)}{p_{3} \Gamma\left(1+p_{1}-\left|q_{3}\right|\right) \Gamma\left(p_{1}-\sigma\right)} K_{\sigma+2}\left(p_{3}\right)
\end{aligned}
$$

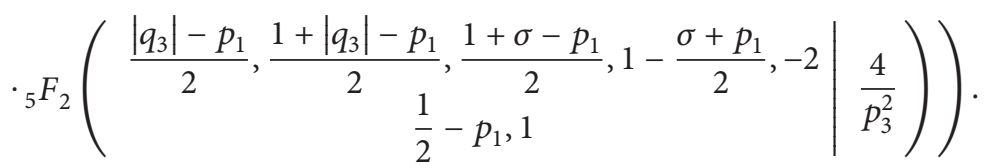


It should be remarked in passing that the same reasoning can be applied to any values of $p_{1}-\left|q_{3}\right|$.

\section{Composition of Basis Transformations and a Representation of the Macdonald Function}

Let $k_{\left(p_{3}, q_{3}\right),\left(p_{2}, q_{2}, \pm\right)}$ be the matrix element of the linear operator acting in $\mathfrak{D}_{\sigma}$ and mapping the hyperbolic basis into the parabolic basis; that is,

$$
f_{p_{3}, q_{3}}^{* *}(x)=\sum_{q_{2} \in \mathbb{Z}} \int_{0}^{+\infty} k_{\left(p_{3}, q_{3}\right),\left(p_{2}, q_{2}, \pm\right)} f_{p_{2}, q_{2}, \pm}^{*}(x) \mathrm{d} p_{2} .
$$

The linear operator $\left(d_{\left(p_{3}, q_{3}\right),\left(p_{1}, q_{1}\right)}\right)$ can be represented as the composition of the linear operators $\left(c_{\left(p_{2}, q_{2}, \pm\right),\left(p_{1}, q_{1}\right)}\right)$ and $\left(k_{\left(p_{3}, q_{3}\right),\left(p_{2}, q_{2}, \pm\right)}\right)$; namely,

$$
\left(d_{\left(p_{3}, q_{3}\right),\left(p_{1}, q_{1}\right)}\right)=\left(k_{\left(p_{3}, q_{3}\right),\left(p_{2}, q_{2}, \pm\right)}\right)\left(c_{\left(p_{2}, q_{2}, \pm\right),\left(p_{1}, q_{1}\right)}\right) .
$$

From this equality, we have, in particular,

$$
\begin{aligned}
& d_{\left(p_{3}, q_{3}\right),\left(\left|q_{3}\right|,-q_{3}\right)} \\
& \quad=\int_{0}^{+\infty} k_{\left(p_{3}, q_{3}\right),\left(p_{2}, q_{3}, \pm\right)} \mathcal{C}_{\left(p_{2}, q_{3}, \pm\right),\left(\left|q_{3}\right|,-q_{3}\right)} \mathrm{d} p_{2} .
\end{aligned}
$$

Theorem 6. The following formula holds true: for $\mathfrak{R}(\sigma)<$ $-(1 / 2)$,

$$
\begin{aligned}
& K_{\sigma+1}\left(p_{3}\right) \\
& =2 \pi p_{3}^{\sigma} \Gamma(-\sigma) \sum_{n=0}^{\infty} \frac{(-1)^{n}}{2^{2 n+1}[n !]^{2}} B(n+1,-2 \sigma-1) \\
& \quad \times{ }_{2} F_{1}(n+1,-\sigma ; n-2 \sigma ;-1) .
\end{aligned}
$$

Proof. Note that the restrictions of the functions $f_{p_{2}, q_{2}, p m}^{*} \in$ $\mathfrak{D}_{-\sigma-2}$ and $f_{p_{3}, q_{3}}^{* *} \in \mathfrak{D}_{\sigma}$ to $\gamma_{3}$ are, respectively,

$$
\begin{aligned}
& \left.f_{p_{2}, q_{2}, p m}^{*}\right|_{\gamma_{2}} \\
& \quad \equiv f_{p_{2}, q_{2}, p m}^{*}\left(\alpha_{3}, \beta_{3}\right) \\
& \quad=2^{\sigma+2}\left(1-\alpha_{3}^{2}\right)_{ \pm} P_{-(1 / 2)+\mathbf{i} p_{2}}^{-\left|q_{2}\right|}\left(\frac{1+\alpha_{3}^{2}}{1-\alpha_{3}^{2}}\right) \exp \left(\mathbf{i}\left|q_{2}\right| \beta_{3}\right), \\
& \left.f_{p_{3}, q_{3}}^{* *}\right|_{\gamma_{2}} \equiv f_{p_{3}, q_{3}}^{* *}\left(\alpha_{3}, \beta_{3}\right)=J_{\left|q_{3}\right|}\left(\alpha_{3} p_{3}\right) \exp \left(\mathbf{i}\left|q_{3}\right| \beta_{3}\right) .
\end{aligned}
$$

Setting $q_{3}=0$, we can rewrite (45) as

$$
\begin{gathered}
K_{\sigma+1}\left(p_{3}\right) \\
=2^{-\sigma-1} \sqrt{\pi} p_{3}^{\sigma} \\
\cdot \int_{0}^{+\infty} p_{2} \sinh \left(\pi p_{2}\right) \Gamma\left(\frac{1}{2}+\mathbf{i} p_{2}\right) \Gamma\left(\frac{1}{2}-\mathbf{i} p_{2}\right) \\
\quad \times \Gamma\left(\frac{-\sigma+\mathbf{i} p_{2}}{2}-\frac{1}{4}\right) \Gamma\left(\frac{-\sigma-\mathbf{i} p_{2}}{2}-\frac{1}{4}\right) \\
\cdot \int_{0}^{1} \alpha_{3}\left(1-\alpha_{3}^{2}\right)^{-\sigma-2} J_{0}\left(p_{3} \alpha_{3}\right) P_{-(1 / 2)+\mathbf{i} p_{2}}^{0} \\
\quad \times\left(\frac{1+\alpha_{3}^{2}}{1-\alpha_{3}^{2}}\right) \mathrm{d} \alpha_{3} \mathrm{~d} p_{2} .
\end{gathered}
$$

Changing the order of integration and using formula (see, e.g., [15, Entry 2.17.27-9])

$$
\begin{aligned}
& \int_{0}^{+\infty} x \sinh (\pi n x) \Gamma\left[\frac{1}{2}-\mu+\mathbf{i}, \frac{1}{2}-\mu-\mathbf{i}, v+\mathbf{i} r x, \nu-\mathbf{i} r x\right] \\
& \times P_{\mathbf{i} x-(1 / 2)}^{\mu}(c) \mathrm{d} x=R_{n, r} \quad\left(\Re(\mu) \leq \frac{1}{2} ; \Re(\nu) \geq 0\right) \\
& R_{1,(1 / 2)}= 2^{(3 / 2)-2 v} \pi^{3 / 2} \Gamma\left(2 \nu-\mu+\frac{1}{2}\right) c^{\mu-2 v-(1 / 2)} \\
& \times\left(c^{2}-1\right)^{-\mu / 2}
\end{aligned}
$$

we find, in the case of $\mu=0, r=(1 / 2)$, and $\nu=-(\sigma / 2)-(1 / 4)$, that

$$
\begin{aligned}
K_{\sigma+1}\left(p_{3}\right)= & 2 \pi p_{3}^{\sigma} \Gamma(-\sigma) \\
& \times \int_{0}^{1} \alpha_{3}\left(1-\alpha_{3}^{2}\right)^{-2 \sigma-2}\left(1+\alpha_{3}^{2}\right)^{\sigma} J_{0}\left(p_{3} \alpha_{3}\right) \mathrm{d} \alpha_{3} .
\end{aligned}
$$

Using here the decomposition

$$
J_{\nu}(z)=\sum_{j=0}^{\infty} \frac{(-1)^{j}}{j ! \Gamma(\nu+j+1)}\left(\frac{z}{2}\right)^{\nu+2 j},
$$

we obtain

$$
\begin{aligned}
K_{\sigma+1} & \left(p_{3}\right) \\
= & 2 \pi p_{3}^{\sigma} \Gamma(-\sigma) \\
& \times \sum_{n=0}^{\infty} \frac{(-1)^{n}}{2^{2 n}[n !]^{2}} \int_{0}^{1} \alpha_{3}^{2 n+1}\left(1-\alpha_{3}^{2}\right)^{-2 \sigma-2}\left(1+\alpha_{3}^{2}\right)^{\sigma} \mathrm{d} \alpha_{3} .
\end{aligned}
$$

To evaluate the right-sided integral, considering new variable $\alpha_{3}:=s$, we rewrite it as

$$
\frac{1}{2} \int_{0}^{1} s^{n}(1-s)^{-2 \sigma-2}(1+s)^{\sigma} \mathrm{d} s
$$


and use a known formula (see, e.g., [16, page 315, Entry 3.1978])

$$
\begin{aligned}
\int_{0}^{a} & x^{a-1}(a-x)^{\omega-1}(x+z)^{-\rho} \mathrm{d} x \\
= & a^{\alpha+\omega-1} z^{-\rho} B(\alpha, \omega)_{2} F_{1}(\alpha, \rho ; \alpha+\omega ;-a / z) \\
& (|\arg z|<\pi ; a>0 ; \mathfrak{R}(a)>0 ; \mathfrak{R}(\omega)>0) .
\end{aligned}
$$

This completes the proof.

\section{Conflict of Interests}

The authors declare that there is no conflict of interests regarding the publication of this paper.

\section{Acknowledgments}

The authors would like to express their deep thanks for the reviewers' helpful and critical comments to improve this paper. The first-named author is supported by the Ministry of Education and Science of the Russian Federation (1.8551.2013). The second-named author was supported by Dongguk University (Gyeongju) Research Fund.

\section{References}

[1] I. A. Shilin and J. Choi, "Certain connections between the spherical and hyperbolic bases on the cone and formulas for related special functions," Integral Transforms and Special Functions, 2013.

[2] I. A. Shilin, " $S O(2,1)$-integrals and formulas for Whittaker functions," Russian Mathematics, vol. 56, pp. 47-56, 2012.

[3] I. Shilin, "SO(2,1)-invariant double integral transforms and formulas for the Whittaker functions," Mathematica Balkanica, vol. 26, no. 1-2, pp. 241-256, 2012.

[4] I. A. Shilin and J. Choi, "Formulas for some cylinder functions associatedwith the group $S O(2,1)$," submitted.

[5] N. J. Vilenkin, Special Functions and the Theory of Group Representations, AMS, Providence, RI, USA, 1968.

[6] E. D. Rainville, Special Functions, Macmillan Company, New York, NY, USA, 1960, Reprinted by Chelsea Publishing Company, Bronx, New York, NY, USA, 1971.

[7] A. P. Prudnikov, Yu. A. Brychkov, and O. I. Marichev, Integrals and Series, Vol. 3: Special Functions, OPA (Overseas Publishers Association), Amsterdam B. V. Published under the License of Gordon and Breach Science Publishers, 1990.

[8] I. A. Shilin and A. I. Nizhnikov, "Some formulas for Legendre functions induced by the Poisson transform," Acta Polytech, vol. 51, pp. 70-73, 2011.

[9] I. A. Shilin and A. I. Nizhnikov, "Some formulas for Legendre functions related to the Poisson transform and Lorentz group representation," Journal of Physics: Conference Series, vol. 346, Article ID 012020, 2012.

[10] M. M. Rodrigues, N. Vieira, and S. Yakubovich, "A convolution operator related to the generalized Mehler-Fock and Kontorovich-Lebedev transforms," Results in Mathematics, vol. 63, no. 1-2, pp. 511-528, 2013.

[11] S. B. Yakubovich, Index Transforms, World Scientific, 1996.
[12] H. M. Srivastava and J. Choi, Zeta and q-Zeta Functions and Associated Series and Integrals, Elsevier Science, Amsterdam, The Netherlands, 2012.

[13] Y. A. Brychkov, Handbook of Special Functions, CRC Press, Taylar \& Francis Group, A Chapman \& Hall Book, 2008.

[14] A. Erdélyi, W. Magnus, F. Oberhettinger, and F. G. Tricomi, Higher Transcendental Functions, vol. 2, McGraw-Hill Book Company, New York, NY, USA, 1953..

[15] A. P. Prudnikov, Yu. A. Brychkov, and O. I. Marichev, Integrals and Series, Vol. 2: Special Functions, OPA (Overseas Publishers Association), Amsterdam B. V. Published under the License of Gordon and Breach Science Publishers, 1986.

[16] I. S. Gradshteyn and I. M. Ryzhik, Tables of Integrals, Series, and Products, Academic Press, New York, NY, USA, 1980, Edited by A. Jeffrey, 6th edition, 2000. 


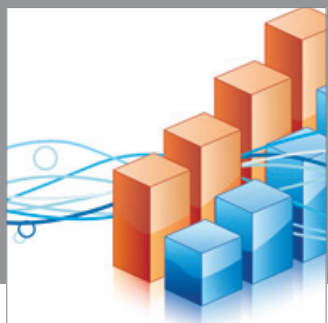

Advances in

Operations Research

mansans

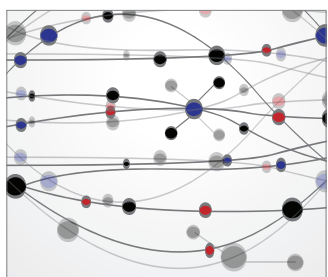

The Scientific World Journal
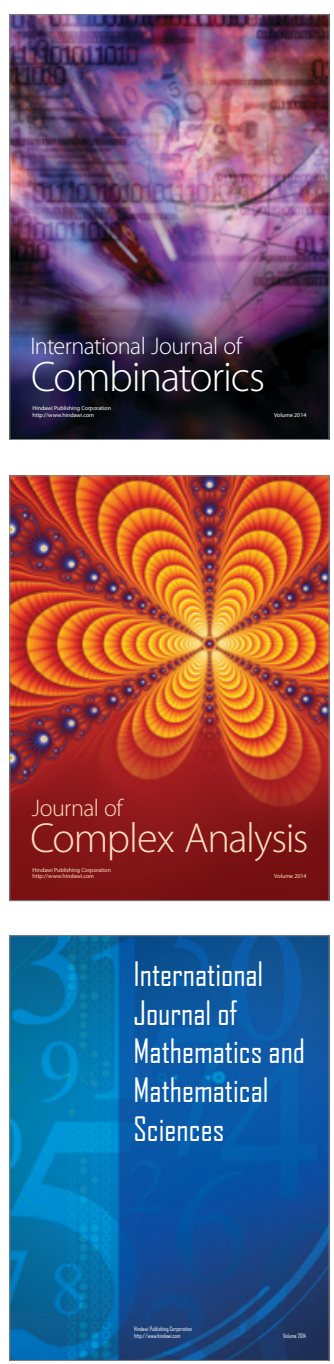
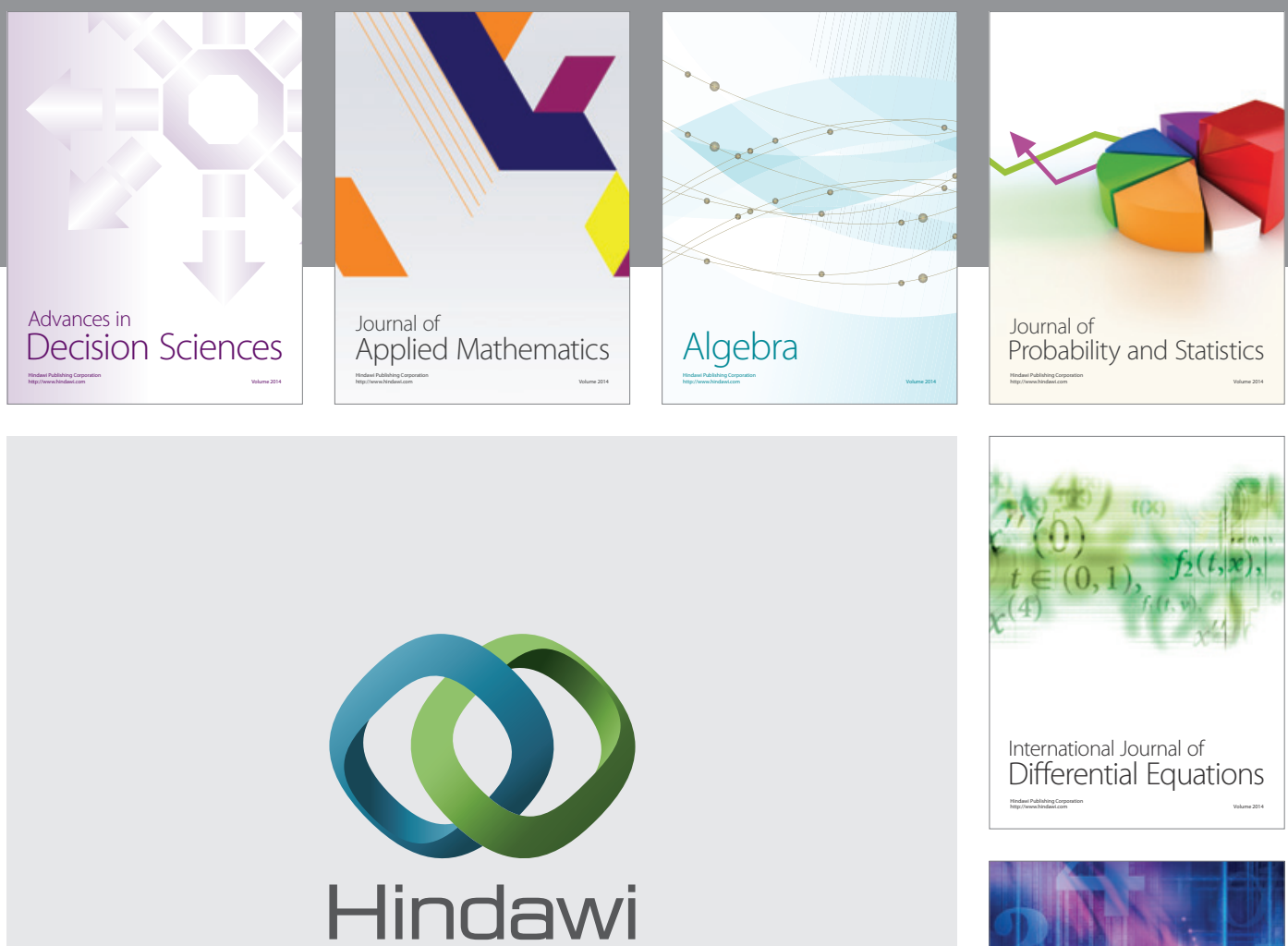

Submit your manuscripts at http://www.hindawi.com
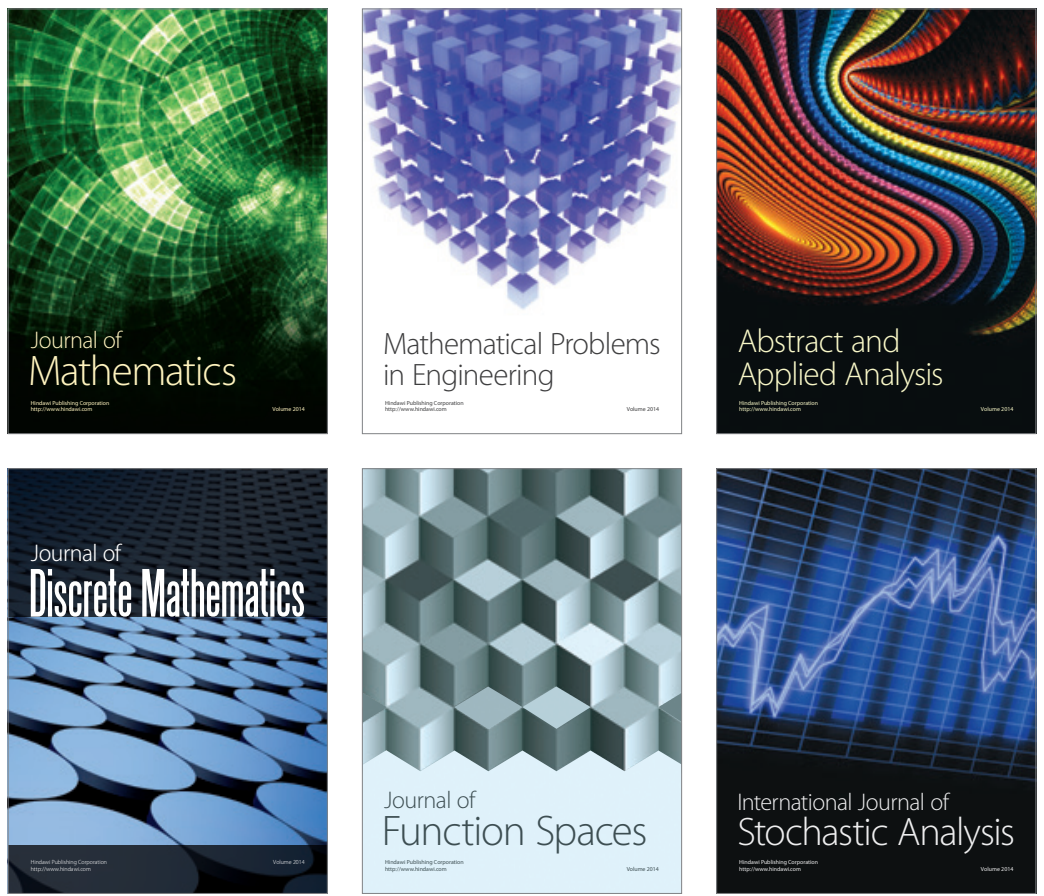

Journal of

Function Spaces

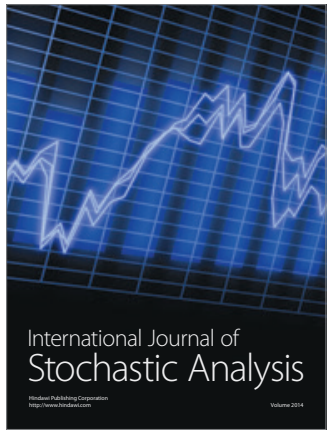

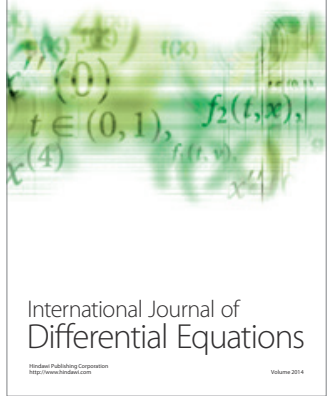
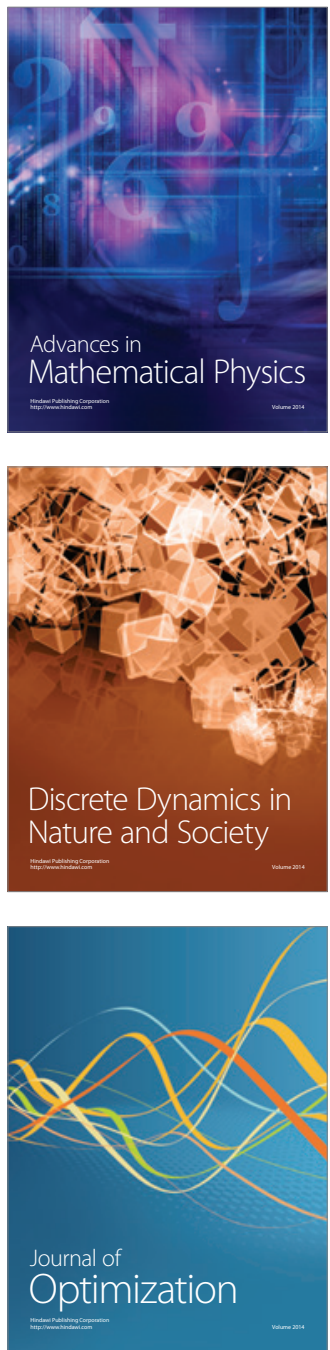